\title{
Toward Deducing the 3-D Thermodynamic Structure from Only Wind Fields - A Validation Study using Simulated Dataset
}

\author{
Yu-Chieng Liou ${ }^{1, *}$
}

(Manuscript received 17 April 2000, in final form 10 December 2000)

\begin{abstract}
This paper presents a newly designed thermodynamic retrieval algorithm whereby one can deduce the potential temperature and pressure gradient fields in a three dimensional space using only 3-D wind measurements. The latter can be observed by Doppler radar in real cases. In order to achieve this purpose, all three equations of motion are implemented in a single cost function. Then, given the detailed wind information, and through the process of variational minimization, these equations are solved simultaneously in a least square sense. The products of the approximated solutions are the three dimensional potential temperature, and the pressure gradients along any direction. Some preliminary studies using model-generated data sets show that this is a feasible tool for the retrievals, and satisfactory results can be obtained when the performance of this method against various types of input data errors is investigated. Compared to the traditional method proposed by Gal-Chen, the advantage of this scheme is that the vertical structure of the thermodynamic variables can be determined without requiring a point of independent observation of the pressure and temperature for each layer. In addition, this method can easily be applied to an area with topography. It is believed that this algorithm can be of particularly useful in many Doppler weather radar applications.
\end{abstract}

(Key words: Thermodynamic retrieval, Variational minimization, Doppler radar)

\section{INTRODUCTION}

It is known that although Doppler radar can provide information about wind with a fine spatial and temporal resolution, the structure of thermodynamic variables, such as temperature and pressure, cannot be directly detected. The pioneer work by Gal-Chen (1978, hereafter GC) demonstrated that given the detailed Doppler radar derived 3-D wind observations, and their

\footnotetext{
${ }^{1}$ Department of Atmospheric Sciences, National Central University, Chung-Li, Taiwan, ROC

${ }^{*}$ Corresponding author address: Dr. Yu-Chieng Liou, Department of Atmospheric Sciences, National Central University, Chung-Li, 320 Taiwan, ROC; E-mail: tyliou@ rainbow.atm.ncu.edu.tw
} 
time derivatives through consecutive volume scans, one could extract the information of the pressure and temperature perturbations. This powerful method turned out to be particularly popular, and was later widely adopted by many researchers to deal with various weather phenomena (e.g., Hane et al. 1981; Gal-Chen and Kropfli 1984; Hane and Ray 1985; Lin et al. 1986; Parsons et al. 1987, among others). Despite the encouraging results, it should be emphasized that GC only retrieves the deviations (from the horizontal mean) of the pressure and temperature perturbations (with respect to a base state) for each horizontal plane. That is, only $\theta^{\prime}-<\theta^{\prime}>$ and $p^{\prime}-<p^{\prime}>$ are solved uniquely at each horizontal layer, where $<>$ stands for a spatial average over that layer. In order to specify the values of $\left\langle\theta^{\prime}\right\rangle$ and $\left\langle p^{\prime}\right\rangle$, and determine the pressure and temperature fluctuations $\theta^{\prime}$ and $p^{\prime}$, at least one point of independent field measurement of the temperature and pressure for each altitude must be available. Although a sounding may provide such information, unfortunately, considering the scenario of daily operations, difficulties persist. First, one has to synchronize the sounding operation with the radar observations. But it is known that the former is usually conducted only twice a day. Secondly, the trace of the sounding may not always remain within the region of the radar volume scans. The consequence of this drawback is that, whenever an interpretation of the vertical structure of the thermodynamic fields is required, one must proceed with caution.

A new approach was proposed by Roux $(1985,1988)$, whereby the pressure and temperature perturbations can be solved uniquely up to a single constant. To deduce this unknown constant requires only one independent pressure and temperature observation at a single point somewhere in the retrieval domain. Roux and Sun (1990) modified the method by Roux (1988) by taking into account a simplified form of the thermodynamic equation everywhere in the domain, so that the temperature gradient was provided both horizontally and vertically. Additional improvements in Roux's retrieval technique can be found in Roux et al. (1993). On the other hand, with the rapid development of the adjoint method, Sun and Crook (1996) demonstrated the advantages of using the so-called 4D-Var technique over the raditional GC scheme for retrieving the thermodynamic field. In contrast to the previous techniques, an important feature shown by the 4D-Var formulation is that the temperature perturbations can be inferred without the auxiliary of any extra, independent measurements.

In this research, a different approach is suggested. The basic methodology involves implementing the horizontal and vertical equations of motion into a single cost function. Through the method of variational analysis, a set of optimally-determined potential temperature fluctuations and pressure perturbation gradients, that minimize this cost function, can be deduced simultaneously.

This paper is organized as follows. In the next section the proposed method is discussed in detail. Section 3 gives the data sets used for verification. Comparisons and discussions of the retrieval results are presented in Section 4, followed by the conclusions in Section 5.

\section{BASIC FORMULATION OF THE RETRIEVAL SCHEME}

In order to fully investigate the performance and properties of this method, the validation is carried out using artificial data sets generated by a numerical model. To simplify the problem, 
the air is assumed to be dry. The basic momentum equations may be written as follows:

$$
\begin{aligned}
& \frac{1}{\theta_{0}}\left[\frac{D u}{D t}-f v+\operatorname{turb}(u)\right]=-\frac{\partial \pi^{\prime}}{\partial x} \equiv-F, \\
& \frac{1}{\theta_{0}}\left[\frac{D v}{D t}+f u+\operatorname{turb}(v)\right]=-\frac{\partial \pi^{\prime}}{\partial y} \equiv-G, \\
& \frac{1}{\theta_{0}}\left[\frac{D w}{D t}+\operatorname{turb}(w)\right]=-\frac{\partial \pi^{\prime}}{\partial z}+g \frac{\theta^{\prime}}{\theta_{0}^{2}} \equiv-H .
\end{aligned}
$$

The Lagrangian time derivative is defined as:

$$
\frac{D}{D t} \equiv \frac{\partial}{\partial t}+u \frac{\partial}{\partial x}+v \frac{\partial}{\partial y}+w \frac{\partial}{\partial z}
$$

where $(u, v, w)$ represent three Cartesian wind components, $\theta$ is the potential temperature, $g$ stands for the gravity, $f$ refers to the Coriolis parameter, and turb () denotes a sub-grid scale turbulence parameterization operator. A normalized form of pressure, called Exner function $\pi$, is used in the model. It is expressed as:

$$
\pi=C_{p}\left(\frac{P}{P_{0}}\right)^{R / C_{p}},
$$

in which $P$ is the pressure, $P_{0}=100 \mathrm{kPa}, R$ is the gas constant, and $C_{p}$ is the specific heat capacity at a constant pressure. The subscript " 0 " represents a horizontally homogeneous basic state, from which the non-hydrostatic perturbations are expressed by variables with a single prime. It can be seen that $F, G$ and $H$ are functions of the winds, as well as the basic state temperature $\theta_{0}$. The three-dimensional air motion can be estimated through either the multiple-Doppler radar synthesis procedure (e.g., Armijo 1969; Ray et al. 1980), or single Doppler wind retrievals (e.g., Sun et al. 1991; Qiu and Xu 1992; Laroche and Zawadzki 1994; Shapiro et al. 1995; Liou 1999, and others), while $\theta_{0}$ can be determined by an environmental sounding.

A cost function can be formulated based on (1)-(3), as shown in the following:

$$
J=\frac{1}{2} \iiint \int_{\Omega}\left(\alpha_{1} P_{1}^{2}+\alpha_{2} P_{2}^{2}+\alpha_{3} P_{3}^{2}+\alpha_{4} P_{4}^{2}+\alpha_{5} P_{5}^{2}+\alpha_{6} P_{6}^{2}\right) d \Omega,
$$

where

$$
\begin{gathered}
d \Omega=d x d y d z d t, \\
P_{1}=\left(\frac{\partial \pi^{\prime}}{\partial x}-F\right), \\
P_{2}=\left(\frac{\partial \pi^{\prime}}{\partial y}-G\right),
\end{gathered}
$$




$$
\begin{aligned}
& P_{3}=\left(\frac{\partial \pi^{\prime}}{\partial z}-g \frac{\theta^{\prime}}{\theta_{0}^{2}}-H\right), \\
& P_{4}=\left(\frac{\partial \theta^{\prime}}{\partial z}\right), \\
& P_{5}=\nabla^{2} \pi^{\prime}, \\
& P_{6}=\nabla^{2} \theta^{\prime}, \\
& \nabla^{2}=\frac{\partial^{2}}{\partial x^{2}}+\frac{\partial^{2}}{\partial y^{2}}+\frac{\partial^{2}}{\partial z^{2}} .
\end{aligned}
$$

In (6), the first two terms $\left(P_{1}^{2}, P_{2}^{2}\right)$ represent the differences between the retrieved horizontal pressure gradients and the calculated $F$ and $G$ (using the Doppler derived 3-D wind observations in real applications), respectively. $P_{3}^{2}$ denotes the difference in the retrieved vertical pressure gradient, the buoyancy, and the quantity $H$. Their weighting coefficients are specified to balance the magnitude of each term. Thus, $\alpha_{1}, \alpha_{2}$, and $\alpha_{3}$ are estimated by:

$$
\begin{aligned}
& \alpha_{1}=\left[\frac{1}{\Omega} \iiint \int_{\Omega}\left(F^{2}\right) d \Omega\right]^{-1}, \\
& \alpha_{2}=\left[\frac{1}{\Omega} \iiint \int_{\Omega}\left(G^{2}\right) d \Omega\right]^{-1}, \\
& \alpha_{3}=\left[\frac{1}{\Omega} \iiint \int_{\Omega}\left(H^{2}\right) d \Omega\right]^{-1},
\end{aligned}
$$

respectively. The weighting coefficient $\alpha_{4}$ is defined as:

$$
\alpha_{4}=\left\{\begin{array}{l}
0.4, \frac{\partial \theta^{\prime}}{\partial z}<0 \\
0.0, \frac{\partial \theta^{\prime}}{\partial z}>0
\end{array} .\right.
$$

Although this coefficient is specified empirically, the general patterns of the retrieved fields are not too sensitive to the value of $\alpha_{4}$. It is known that although the total potential temperature is increasing with height, the vertical gradient of the potential temperature perturbations can be either upward or downward. In other words, $\partial \theta^{\prime} / \partial z<0$ is physically true. However, our experimental results show that this constraint can effectively eliminate the "spurious" thermal instability, while the region of "rue" thermal instability remains intact. Furthermore, 
since $P_{4}^{2}$ is added as a "weak" constraint, therefore, when adequate weighting is given, it would not make $\partial \theta^{\prime} / \partial_{z}<0$ disappear completely. Although interpreted differently, this constraint was also included in the retrieval technique proposed by Roux and Sun (1990), as well as in the improved version suggested by Roux et al. (1993). Sun and Crook (1996) also adopted this constraint for the same purpose in their 4D-Var retrieval formulations.

Finally, constraints $P_{5}^{2}-P_{6}^{2}$ represent low-pass filters to suppress noise. Based on Testud and Chong (1983), the weighting coefficients, $\alpha_{5}$ and $\alpha_{6}$, are estimated by:

$$
\alpha_{5}=\alpha_{6}=\frac{k_{0}^{-4}}{N}=\frac{\mu}{N}
$$

where $k_{0}$ denotes the cutoff wave number, and $N$ is the total number of grid points in the retrieval domain. If $2 \Delta$ is chosen as the cutoff wavelength, where $\Delta$ is the horizontal grid resolution, then the corresponding value of $\mu$ equals $(\Delta / \pi)^{4} \sim 0.01 \Delta^{4}$.

From the first three cost function constraints, it is clear that the potential temperature appears in its undifferentiated form, but the pressure is always expressed by first order derivatives. Consequently, when (6) is minimized, the results will provide the structure of the buoyancy field itself in a three-dimensional space, as well as the pressure fluctuation gradients, not only for each horizontal plane, but also in the vertical direction. In other words, the potential temperature field itself is determined, as is the pressure field up to a "volume wide" constant. No additional information is needed. We believe this to be an important advance over the traditional GC scheme. However, when it is desirable to compare the retrieved pressure perturbations against the true solutions, one should remove the average over the entire domain from each field, respectively.

In order to minimize (6), which is a cost function with very large number of dimensionalities, a quasi-Newton conjugate-gradient algorithm, named VA15AD by Liu and Nocedal (1989), is employed. This method, however, requires the user supplied knowledge of the cost function gradients with respect to $\pi^{\prime}, \theta^{\prime}$ at each point (i.e., $\partial J / \partial \pi^{\prime}$ and $\partial J / \partial \theta^{\prime}$ ). This can be derived by attributing the variation of the cost function, represented by $\delta J$, to the variations of $\pi^{\prime}, \theta^{\prime}$, denoted by $\delta \pi^{\prime}$ and $\delta \theta^{\prime}$ respectively, throughout the interior region as well as over the boundaries. That is:

$$
\delta J=\iiint\left(\frac{\partial J}{\partial \pi^{\prime}} \delta \pi^{\prime}+\frac{\partial J}{\partial \theta^{\prime}} \delta \theta^{\prime}\right) d x d y d z
$$

The readers can refer to Liou (1999) for the details of the derivations. For example, for the interior points, the gradients are expressed by:

$$
\begin{gathered}
\frac{\partial J}{\partial \pi^{\prime}}=\int\left\{-\left[\frac{\partial\left(\alpha_{1} P_{1}\right)}{\partial x}+\frac{\partial\left(\alpha_{2} P_{2}\right)}{\partial y}+\frac{\partial\left(\alpha_{3} P_{3}\right)}{\partial z}\right]\right. \\
\left.+\frac{\partial^{2}}{\partial x^{2}}\left(\alpha_{5} \frac{\partial^{2} \pi^{\prime}}{\partial x^{2}}\right)+\frac{\partial^{2}}{\partial y^{2}}\left(\alpha_{5} \frac{\partial^{2} \pi^{\prime}}{\partial y^{2}}\right)+\frac{\partial^{2}}{\partial z^{2}}\left(\alpha_{5} \frac{\partial^{2} \pi^{\prime}}{\partial z^{2}}\right)\right\} d t,
\end{gathered}
$$




$$
\begin{gathered}
\frac{\partial J}{\partial \theta^{\prime}}=\int\left\{-\left[\frac{\alpha_{3} P_{3} g}{\theta_{0}^{2}}+\frac{\partial\left(\alpha_{4} \frac{\partial \theta^{\prime}}{\partial z}\right)}{\partial z}\right]\right. \\
\left.+\left[\frac{\partial^{2}}{\partial x^{2}}\left(\alpha_{6} \frac{\partial^{2} \theta^{\prime}}{\partial x^{2}}\right)+\frac{\partial^{2}}{\partial y^{2}}\left(\alpha_{6} \frac{\partial^{2} \theta^{\prime}}{\partial y^{2}}\right)+\frac{\partial^{2}}{\partial z^{2}}\left(\alpha_{6} \frac{\partial^{2} \theta^{\prime}}{\partial z^{2}}\right)\right]\right\} d t .
\end{gathered}
$$

Using an initial guess (usually zero) for the unknown variables $\pi^{\prime}$ and $\theta^{\prime}$, and the gradients for $J$ computed by (11)-(12), subroutine VA15AD determines a search direction whereby it finds a new set of $\pi^{\prime}$ and $\theta^{\prime}$ that reduce the value of the cost function $J$. This new solution becomes another initial guess, and the searching process continues until the reduction of the cost function converges to zero. Unlike the traditional GC algorithm in which the spatial derivatives of $\pi^{\prime}$ is specified along the boundaries using wind observations in order to solve a Poisson equation for $\pi^{\prime}$, one advantage of this method is that the boundary conditions for $\pi^{\prime}$ and $\theta^{\prime}$ are not required in the minimization procedure.

All existing thermodynamic retrieval techniques start by using real Doppler radar derived wind data, in which observational errors are already embedded, to estimate those terms on the left hand side of momentum equations shown by (1)-(3) (i.e., the local time rate of change, advection, Coriolis force, and sub-grid parameterizations). The estimations are usually conducted in finite difference form, and it is known that this procedure would inevitably amply the errors. The author would like to point out that the minimization algorithms developed by Roux $(1985,1988)$, and Roux and Sun (1990) require additional spatial derivatives of the momentum equations, and this procedure is likely to further amplify the impacts of the observational errors. By contrast, in this research, the momentum equations are directly applied in their original forms without any further derivatives. Thus, the problem mentioned above can be avoided.

\section{INPUT DATA SETS AND VERIFICATION}

In this paper the data sets utilized for validation come from the numerical simulation of a group of thermal bubbles. The model domain contains a total of $55 \times 55 \times 55$ grid points. The horizontal and vertical resolutions are specified to be $1.0 \mathrm{~km}$ and $0.15 \mathrm{~km}$, respectively. Thus, the simulation is performed within a three-dimensional domain with a volume of approximately $55 \times 55 \times 8 \mathrm{~km}^{3}$. The background atmosphere is assumed to be neutral, with a basic state potential temperature of $300^{\circ} \mathrm{k}$. The temperature perturbations are given at the layer of $\mathrm{Z}$ $=225 \mathrm{~m}$. The formulation of each thermal bubble is expressed by:

$$
\theta^{\prime}(x, y)=\frac{\theta_{m}^{\prime}}{1+\left(\frac{x-x_{c}}{x_{r}}\right)^{2}+\left(\frac{y-y_{c}}{y_{r}}\right)^{2}},
$$


where $\theta_{m}^{\prime}$ denotes the maximum magnitude of the bubble, $\left(x_{c}, y_{c}\right)$ are the coordinates of the bubble's center, and $\left(x_{r}, y_{r}\right)$ represent the half-widths of the perturbation in $\mathrm{x}$ and $\mathrm{y}$ directions respectively. In this study they both are given the value of $10 \mathrm{~km}$. A group of five bubbles is given to the initial temperature perturbation field, with their centers located at $(28 \mathrm{~km}, 28 \mathrm{~km})$, $(14 \mathrm{~km}, 28 \mathrm{~km}),(42 \mathrm{~km}, 28 \mathrm{~km}),(28 \mathrm{~km}, 14 \mathrm{~km})$ and $(28 \mathrm{~km}, 42 \mathrm{~km})$ respectively. The value of $\theta_{m}^{\prime}$ is specified by $3^{\circ} \mathrm{k}$, except for the bubble at $(28 \mathrm{~km}, 28 \mathrm{~km})$, where a stronger $\theta_{m}^{\prime}$ equals to $6^{\circ} \mathrm{k}$ is provided. The model is numerically integrated forward in time for 40 minutes, using leap-frog scheme with a time step $\Delta t$ of 6 seconds. No perturbations are imposed to the initial pressure field. The dimensions of the domain, as well as the size and magnitude of the initial conditions are carefully specified so that the fields of the pressure and temperature fluctuations remain well inside the domain throughout the entire simulation. Figures 1 and 2 show the simulated pressure and potential temperature perturbations on a vertical $\mathrm{x}-\mathrm{z}$ cross section passing through the center of the domain after 35 minutes of simulation time, while their horizontal distributions at the height of $1 \mathrm{~km}$ are illustrated in Figs. 3 and 4. Note that in Figs. 1 and 3, the volume wide average has been removed. These four fields are selected as true solutions for verifying the retrieval results.

In additional to the visual comparisons, the accuracy of the retrieval results is investigated by evaluating several quantitative indices. If " $A$ " represents either the potential temperature or the pressure, and the subscripts " $i$ " or " $r$ " denote the "true" or "retrieved" quantities respectively, we then obtain the following definitions:

Spatial correlation coefficient (SCC) :

$$
\begin{gathered}
S C C(A)=\frac{\sum\left(A_{r}-\bar{A}_{r}\right)\left(A_{t}-\bar{A}_{t}\right)}{\sqrt{\sum\left(A_{r}-\bar{A}_{r}\right)^{2} \sum\left(A_{t}-\bar{A}_{t}\right)^{2}}} \\
\bar{A}=\frac{1}{N} \sum A .
\end{gathered}
$$

Here, $\bar{A}$ can be an average taken either over a three-dimensional volume, or a two-dimensional plane. $N$ is the total number of grid points in the volume/plane.

Reliability parameter for retrievals $\left(E_{r}\right)$ :

$$
\begin{aligned}
& E_{r} \equiv \\
& \frac{\iiint\left[\left(\frac{\partial \pi_{r}^{\prime}}{\partial x}-F\right)^{2}+\left(\frac{\partial \pi_{r}^{\prime}}{\partial y}-G\right)^{2}+\left(\frac{\partial \pi_{r}^{\prime}}{\partial z}-\frac{\partial \pi_{t}^{\prime}}{\partial z}\right)^{2}+\left(g \frac{\theta_{r}^{\prime}}{\theta_{0}^{2}}-g \frac{\theta_{t}^{\prime}}{\theta_{0}^{2}}\right)^{2}\right] d x d y d z}{\iiint\left[F^{2}+G^{2}+\left(\frac{\partial \pi_{t}^{\prime}}{\partial z}\right)^{2}+\left(g \frac{\theta_{t}^{\prime}}{\theta_{0}^{2}}\right)^{2}\right] d x d y d z} .
\end{aligned}
$$




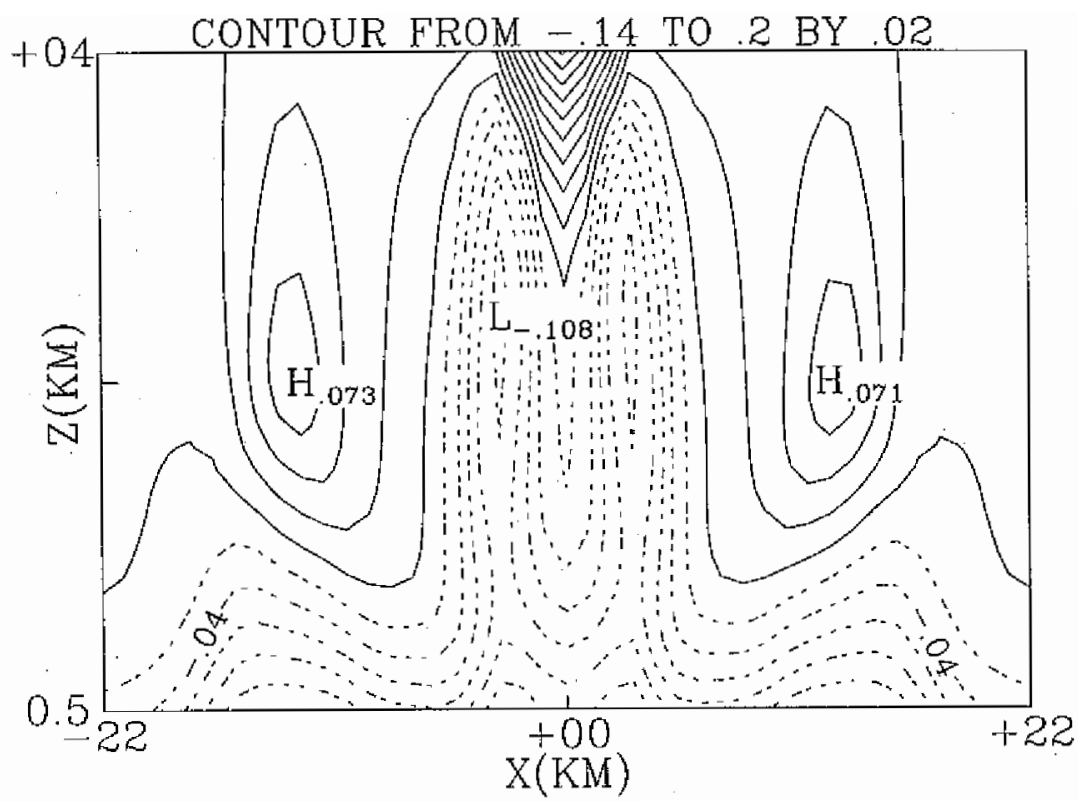

Fig. 1 . The model-generated "true" pressure field on a vertical cross section crossing the center of the domain. The volume wide mean has been removed.

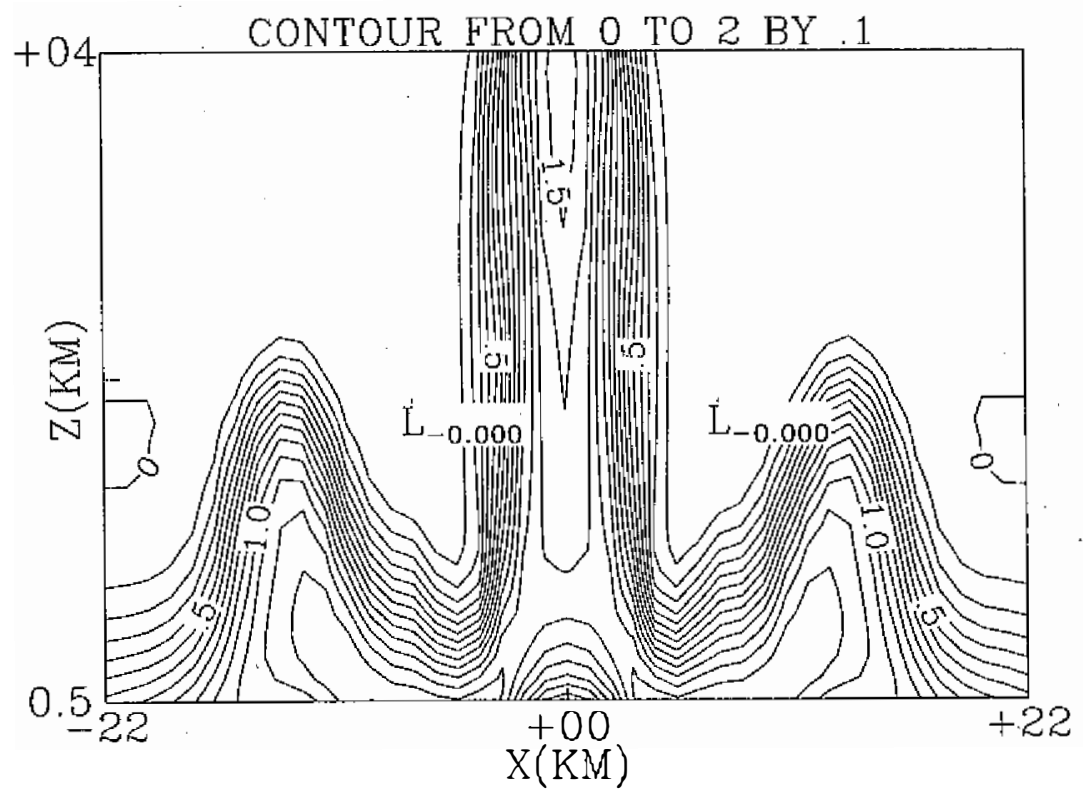

Fig. 2. The model-generated "true" potential temperature field on a vertical cross section crossing the center of the domain. 


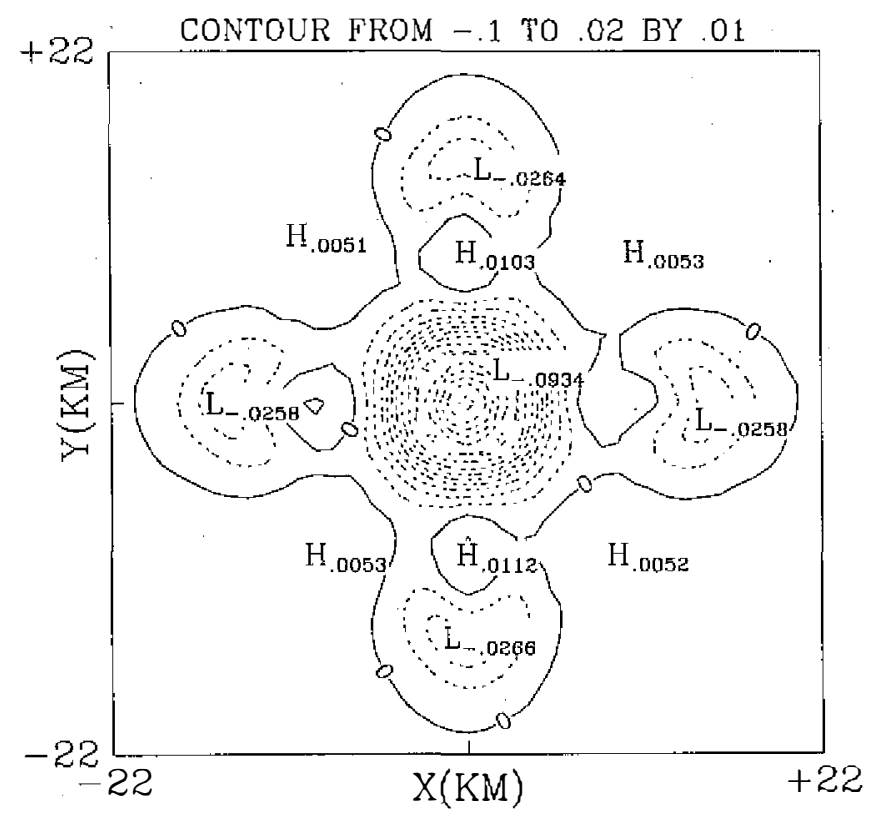

Fig. 3. The model-generated "true" pressure field on a horizontal cross section at $\mathrm{Z}=1 \mathrm{~km}$. The volume wide mean has been removed.

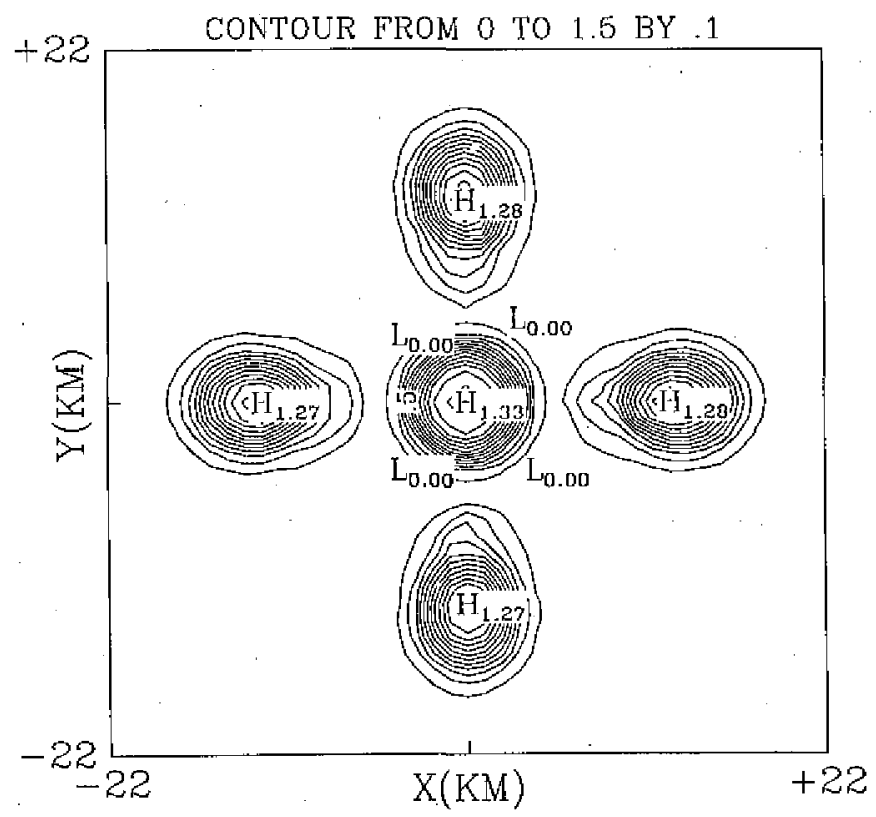

Fig. 4. The model-generated "true" potential temperature field on a horizontal cross section at $Z=1 \mathrm{~km}$. 
The SCC score measures the "similarity" between two fields, and reaches 1 when they are identical. The purpose of calculating this index is that in many diagnostic studies of weather phenomena using Doppler radar data, it suffices to construct the "pattern" of the thermodynamic variables. In other words, one is interested in the relative positions of the high/low pressure, as well as the warm/cold centers, rather than the exact numbers at each grid point. As for the reliability parameter $E_{r}$, it estimates the relative errors of the retrieval, and is analogous to the "momentum-checking" parameter by Gal-Chen and Kropfli (1984). They gave a threshold of 0.5 , above which the retrieval is believed to be virtually meaningless. Hane and Ray (1985) also suggested that an $E_{r} \leq 0.25$ would be considered to be a successful retrieval.

\section{RESULTS}

Most of the retrieval experiments were conducted within a smaller domain of $45 \times 45 \times 33$ grid points in order to avoid the influence from the numerical model's boundary conditions. Only preliminarily investigations are executed in this study. Basically, these experiments are conducted to explore the feasibility of the proposed formulation, as well as its sensitivity to two commonly encountered degraded input data in radar meteorology, namely, the discrete nature of the Doppler radar volume scans, and the wind data with embedded observational errors. We will also focus on the vertical structure of the retrieved thermodynamic variables.

\subsection{Reference Run}

The first experiment represents a reference run in which the tendency terms in the input data sets $F, G$ and $H$ (i.e., $\partial u / \partial t, \partial \nu / \partial t, \partial w / \partial t$ ) are calculated accurately by finite differencing the model outputs of the velocity fields at $T=35 \mathrm{~min}-6 \mathrm{~s}$ and $T=35 \mathrm{~min}+6 \mathrm{~s}$. Note that $6 \mathrm{~s}$ is the length of the leap-frog time step in the model integration. For the advection, Coriolis force, and turbulence parameterizations, we use the model results at $T=35 \mathrm{~min}$. The $F, G$ and $H$ are then substituted into the retrieval algorithm without contamination. Note that even with perfect input data, one should not expect an exact solution from the proposed scheme. This is because the strategy as outlined in Section 2 only iteratively seeks an approximated solution, which will minimize the differences between the momentum equations and the input data. By contrast, the traditional methods, such as GC, directly solve for the exact thermodynamic variables from the model equations. With this in mind, Figs. 5-8 display the retrieved pressure and potential temperature perturbations along the vertical and horizontal planes respectively. Their degree of agreement to the true solutions (Figs. 1-4) is very encouraging. The author would like to emphasize that not only the horizontal distributions of the pressure and temperature are correct, the vertical structures can also be determined with certainty.

To make a comparison quantitatively, the indices introduced in (14)-(15) are calculated globally over the entire retrieval domain. Table 1 shows the resulting statistics. The value of $E_{r}$ $(=0.149)$ is far below 0.25 , indicating a successful retrieval. Of particular satisfaction is the good SCC scores $(>0.9)$ for all retrieved variables, which imply a close similarity between the retrieved and the true fields over a three-dimensional space. The SCC parameters are also estimated for the selected vertical and horizontal layers as displayed in Figs. 5-8, and they are 


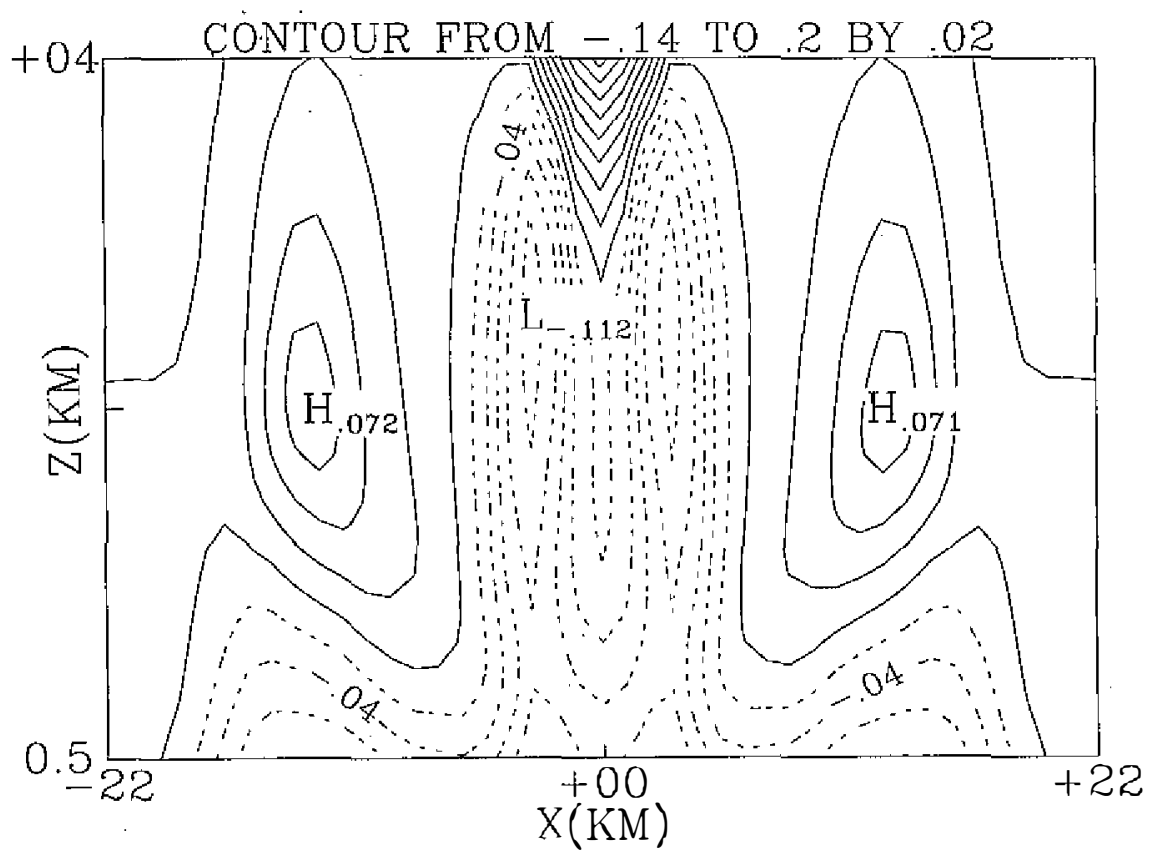

Fig. 5. As in Fig. 1, but for Experiment 1, the reference run. SCC $=0.976$.

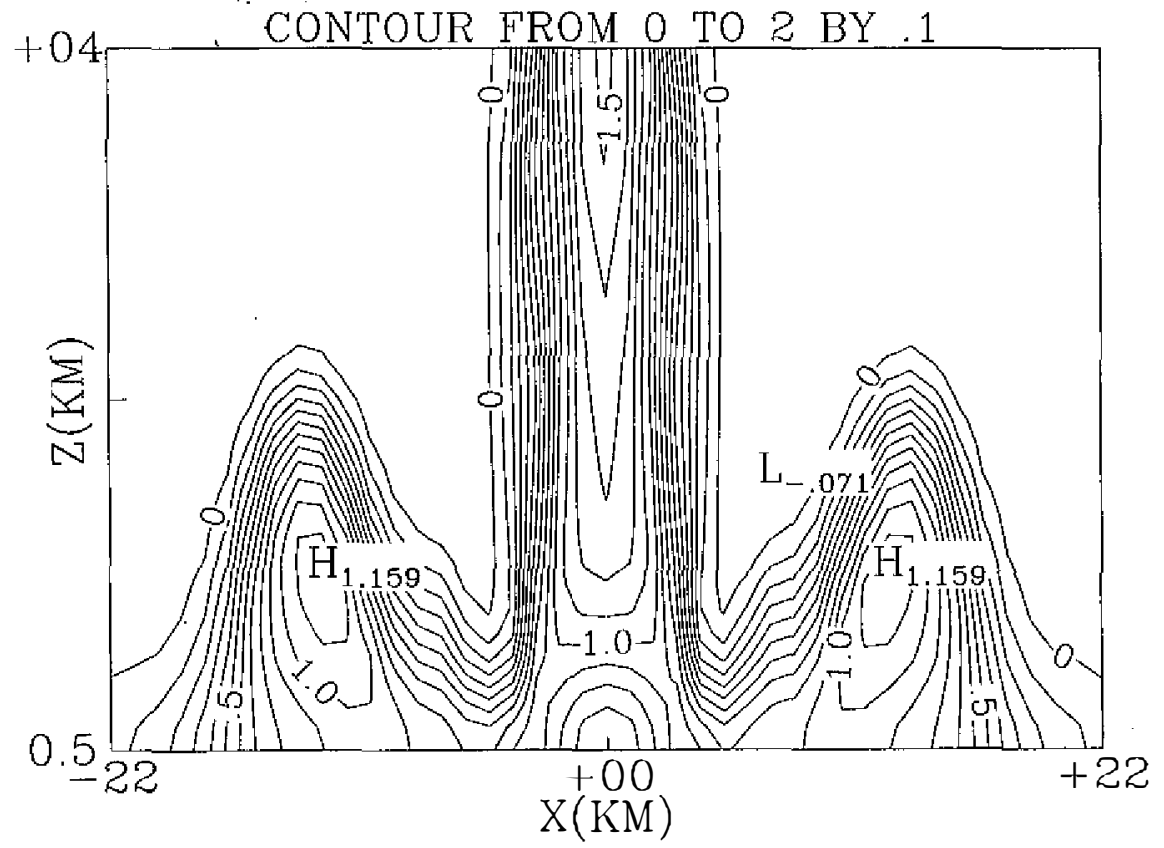

Fig. 6. As in Fig. 2, but for Experiment 1, the reference run. SCC $=0.988$. 


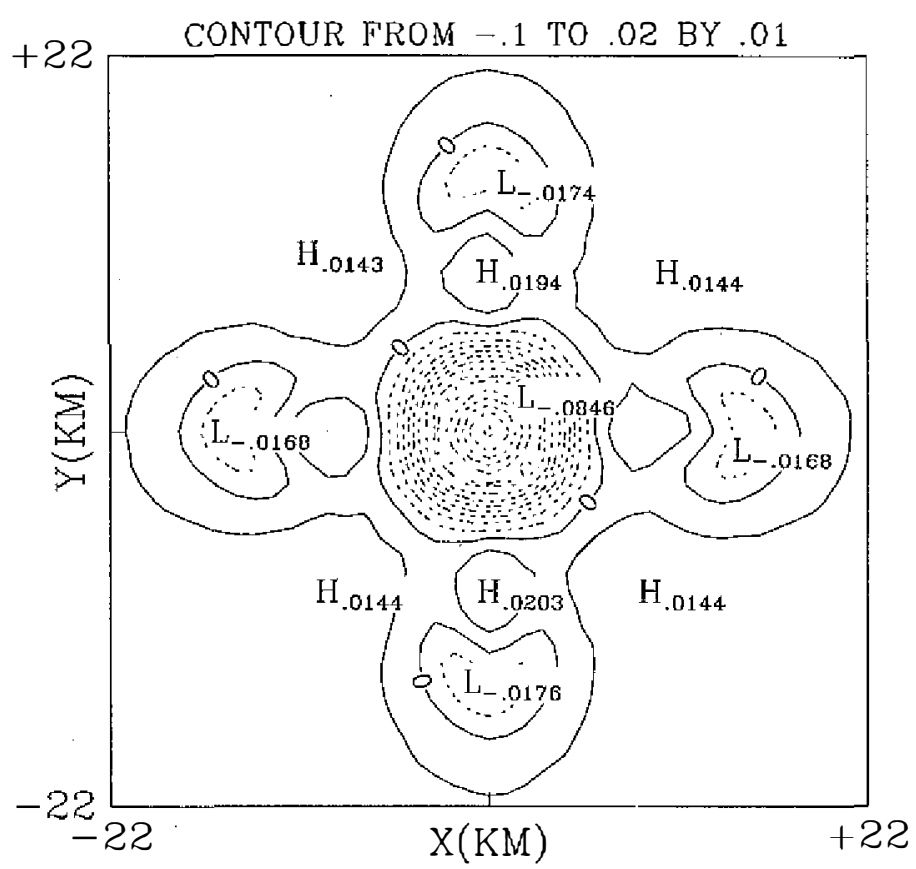

Fig. 7. As in Fig. 3, but for Experiment 1, the reference run. SCC $=0.999$.

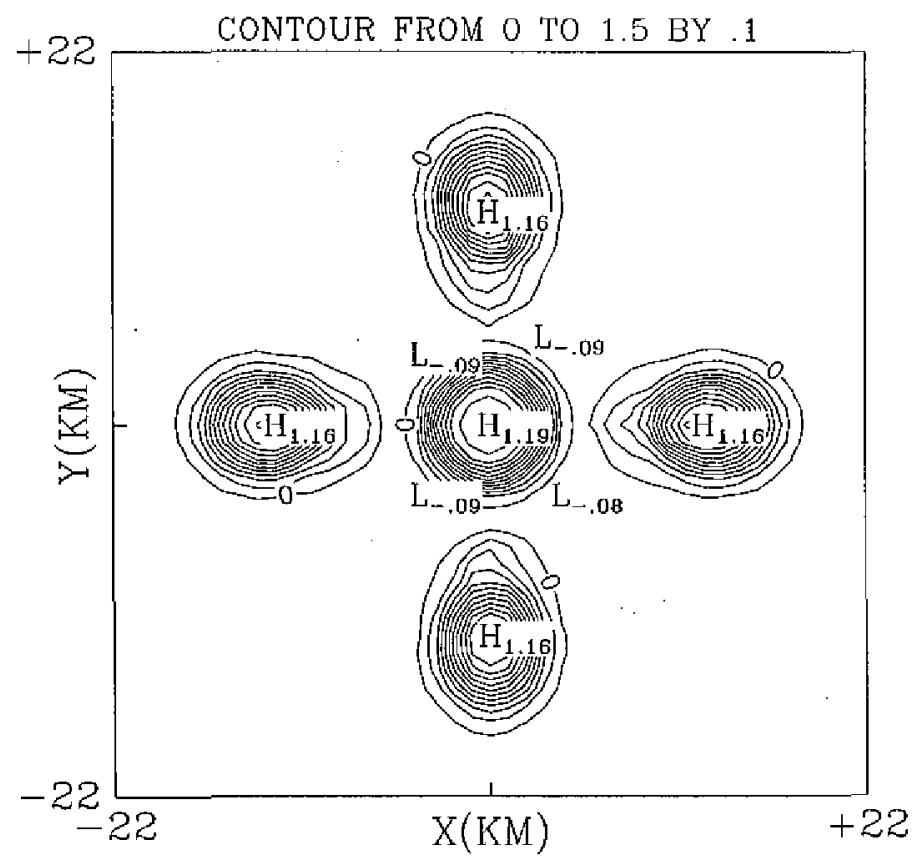

Fig. 8. As in Fig. 4, but for Experiment 1, the reference run. SCC=0.999. 
Table 1. SCC scores and the value of Er for Experiments 1 5.

\begin{tabular}{|c|c|c|c|c|}
\hline \multirow{2}{*}{ Experiment } & \multirow{2}{*}{ Description } & \multicolumn{2}{|c|}{ SCC } & \multirow{2}{*}{$E r$} \\
\hline & & $\pi^{\prime}$ & $\theta^{\prime}$ & \\
\hline 1 & $\begin{array}{l}\text { Testing the feasibility of this method } \\
\text { (Reference nun) }\end{array}$ & 0.903 & 0.967 & 0.149 \\
\hline 2 & $\begin{array}{l}\text { Testing the sensitivity to time intervals } \\
\text { between radar scans }(\Delta t=180 \mathrm{~s})\end{array}$ & 0.901 & 0.941 & 0.199 \\
\hline 3 & $\begin{array}{l}\text { Testing the sensitivity to time intervals } \\
\text { between radar scans }(\Delta t=300 s)\end{array}$ & 0.899 & 0.935 & 0.200 \\
\hline 4 & $\begin{array}{l}\text { Testing the hypothesis of local steady- } \\
\text { state }(\partial / \partial t \equiv 0)\end{array}$ & 0.149 & 0.466 & 1.049 \\
\hline 5 & $\begin{array}{l}\text { Testing the tolerance to observational } \\
\text { errors embedded in wind data }\end{array}$ & 0.895 & 0.960 & 0.188 \\
\hline
\end{tabular}

found to be very close to one. Since this study emphasizes the interpretation of the vertical structures, the SCC indices are computed again for each two dimensional $\mathrm{x}-\mathrm{z}$ cross section. For example, Figure 9 displays the resulting distributions of potential temperature perturbation slice by slice along the y coordinate, and one immediately recognizes that the high scores assure that the vertical structures of thermodynamic perturbations can be determined without ambiguity.

\subsection{Sensitivity to Time Intervals Between Radar Volume Scans}

As discussed in GC, taking the time derivatives over a long time interval is equivalent to simulating errors due to non-simultaneous radar observations. A criteria for an allowable radar scan time $\Delta t$ is suggested in Gal-Chen (1979) by:

$$
\frac{U \Delta t}{L} \sim \mathrm{O}(1),
$$

where $U$ is a typical velocity, and $L$ stands for the spatial resolution. In Experiment 2, the sensitivity of this retrieval scheme to the time intervals between each wind observation is explored. As mentioned earlier in the previous section, the tendency terms (i.e., $\partial u / \partial t, \partial v / \partial t$, $\partial w / \partial t$ ) in the input data sets $F, G, H$ are prepared by finite differencing the model outputs of the velocity fields at $T=35 \mathrm{~min}-\Delta t$ and $T=35 \mathrm{~min}+\Delta t$, where $\Delta t$ is the length of the leapfrog time step. For the advection, Coriolis force, and turbulence parameterizations, they are evaluated using the model-produced wind outputs at $T=35 \mathrm{~min}$. In this experiment, we prolong the time intervals between two radar data sets gradually from 12s (Experiment 1), to 180s (Experiment 2), and to 300s (Experiment 3). Note that some research Doppler radar (e.g., 


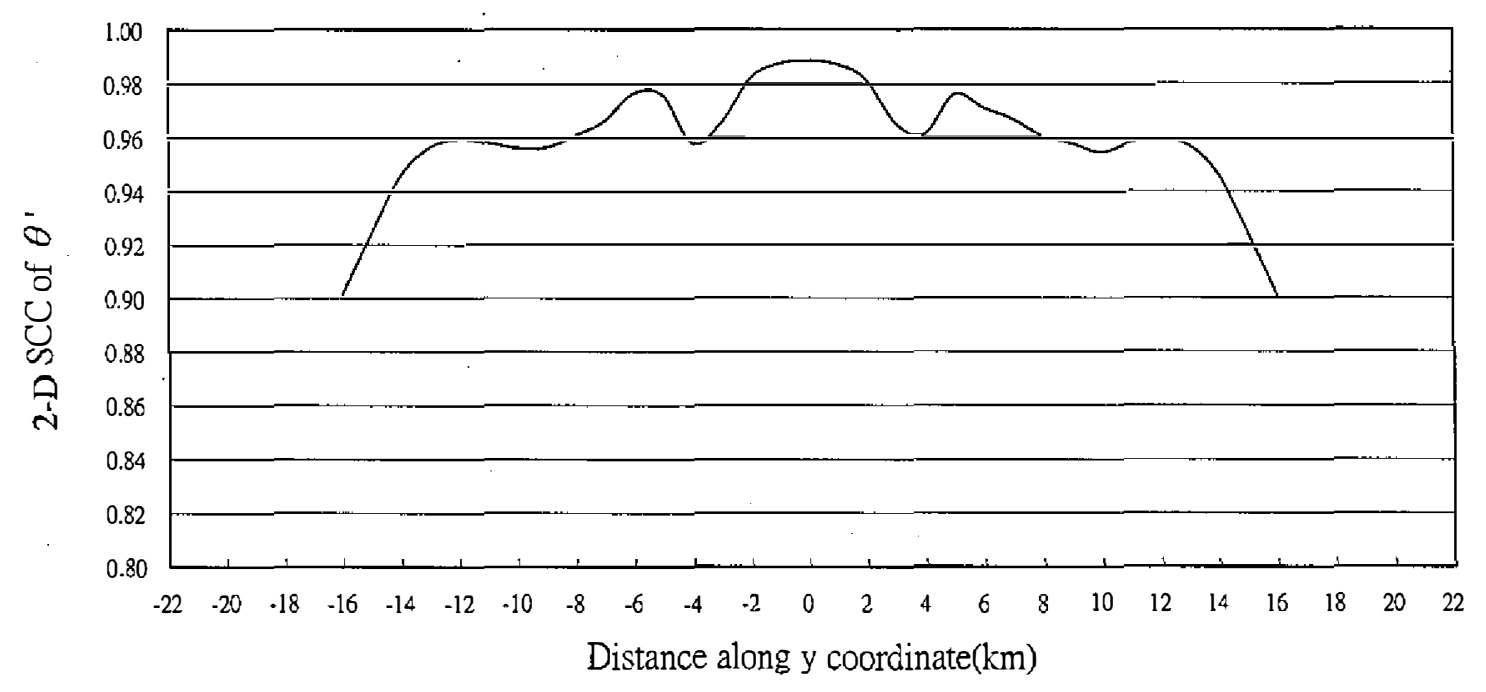

Fig. 9. The 2-D SCC scores of potential temperature perturbations $\left(\theta^{\prime}\right)$ on each $\mathrm{x}-\mathrm{z}$ cross section along the y coordinate for Experiment 1.

Doppler on Wheel) is capable of conducting 60s to 180 s fast scans, while 300 s is approximately the time interval between WSR-88D volume scans under operational mode.

Table 1 lists the results of the quantitative comparisons. It is obvious that the proposed method works very well as $\Delta t$ increases from $12 \mathrm{~s}$ to $300 \mathrm{~s}$. The magnitude of $E_{r}$ varies from 0.149 to 0.200 , but still under the threshold value of 0.25 . It may also be noted that in this experiment the SCC scores for both pressure and temperature remain close or above 0.90 for different time intervals, which implies, at least qualitatively, that the retrieved thermodynamic perturbation fields are robust for this kind of degraded data. The vertical and horizontal structure of the retrieved pressure and temperature in Experiment 3 are illustrated in Figs. 10-13, along with the calculated SCC scores for that particular slice of the domain. The agreements with their counterparts shown in Figs. 1-4 are very satisfactory. Therefore, Experiments 2 and 3 state that this retrieval algorithm does have a certain tolerance to the errors associated with the discreteness of the radar measurements. In real case applications, one should realize that the importance of the time derivative term is case dependent, but the author believes that an intensive scanning strategy would be beneficial in terms of improving the quality of the retrievals.

In Experiment 4 the time derivatives are completely dropped out from the calculations of $F, G$, and $H$ to test the validity of local steady-state assumption. From Table 1 it can be seen that the quality of the retrievals deteriorates severely, as is shown by the value of $E_{r}$ which exceeds the threshold, and becomes 1.049. Such a large number indicates that this revieval is virtually a failure. Significant differences are also found in the plots of the retrieved fields. For example, Figure 14 denotes the potential temperature perturbations on the same $\mathrm{x}-\mathrm{z}$ cross section as in Fig. 2, but the SCC score is found to be as low as 0.476 . One should realize that the result found in this experiment is in agreement with that from GC, in which the commonly 


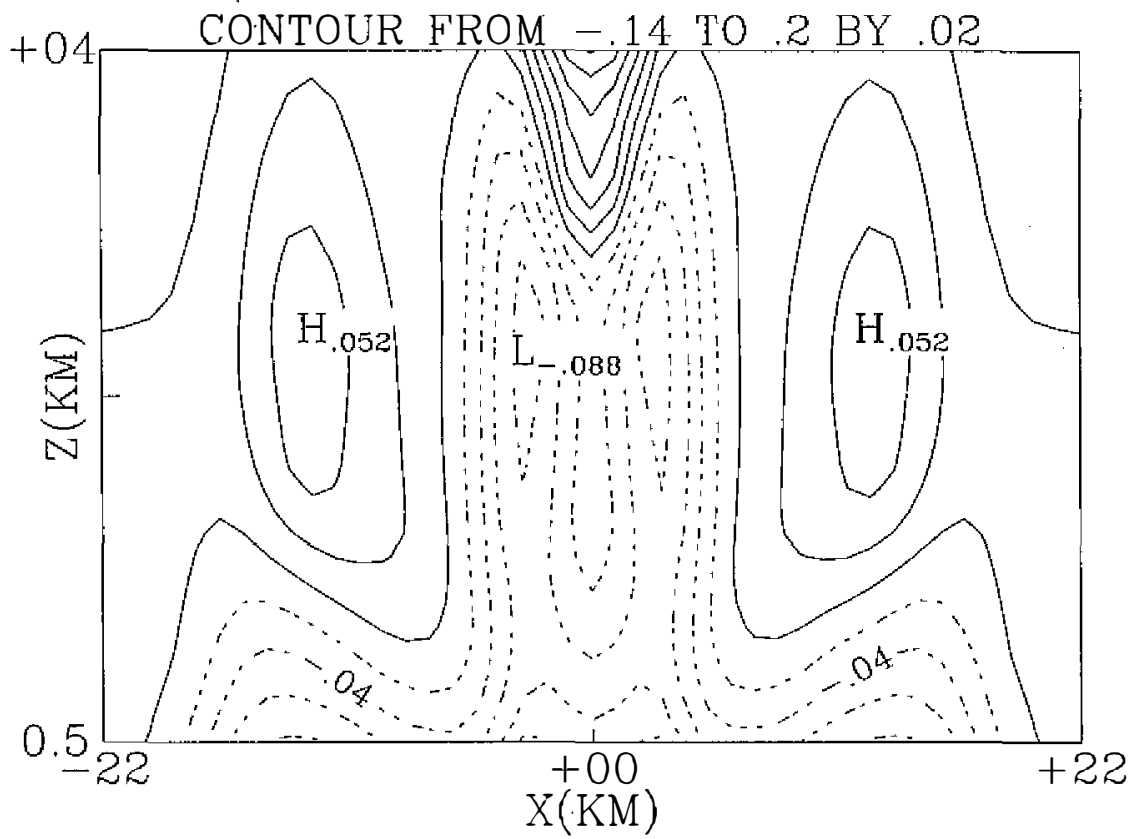

Fig. 10. As in Fig. 1, but for Experiment 3 with $\Delta t=300$ s. SCC $=0.972$.

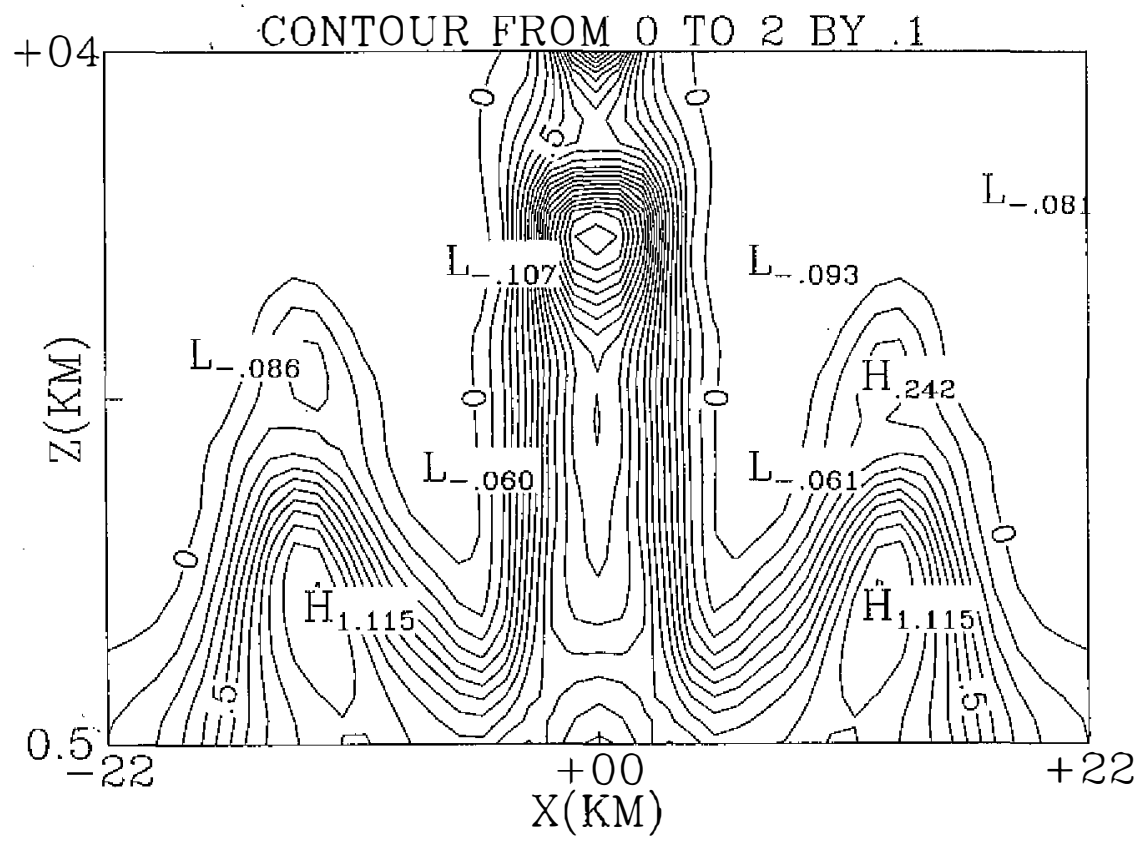

Fig. 11. As in Fig. 2, but for Experiment 3 with $\Delta t=300 \mathrm{~s}$. SCC $=0.927$. 


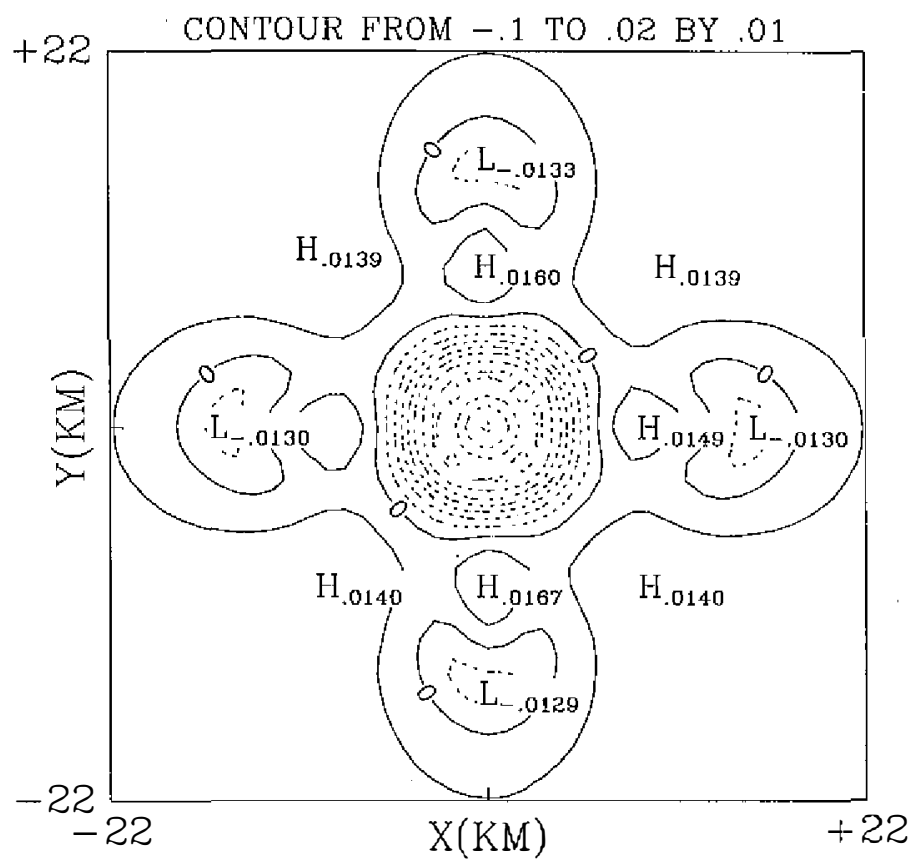

Fig. 12. As in Fig. 3, but for Experiment 3 with $\Delta t=300 \mathrm{~s} . \mathrm{SCC}=0.999$.

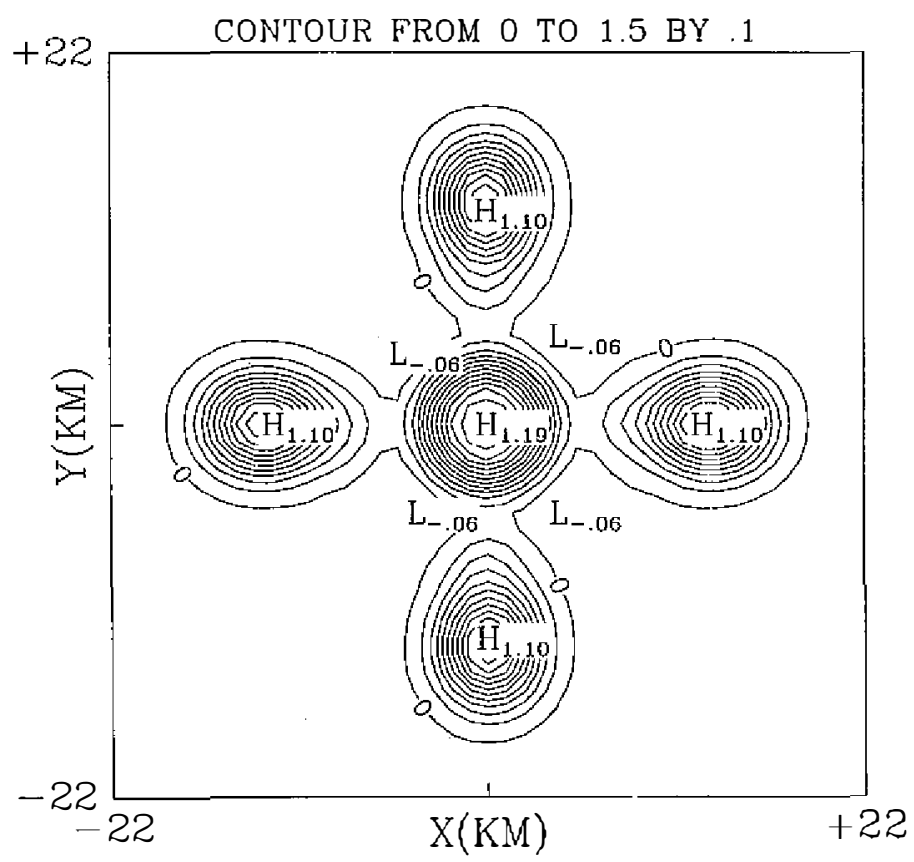

Fig. 13. As in Fig. 4, but for Experiment 3 with $\Delta t=300 \mathrm{~s}$. SCC $=0.998$. 


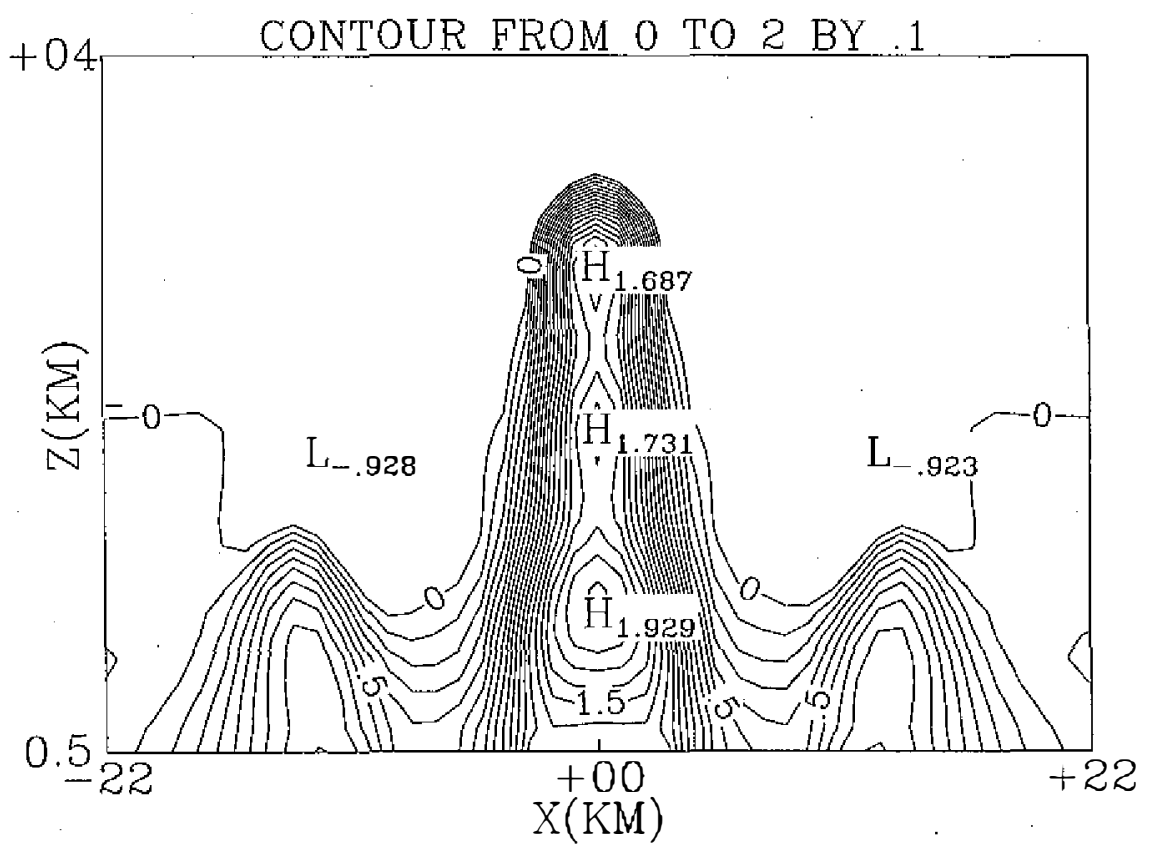

Fig. 14. As in Fig. 2, but for Experiment 4 with the tendency terms neglected. $\mathrm{SCC}=0.476$

used local steady-state hypothesis was actually disqualified. In additional, GC also suggested that the meaning of steady-state should be interpreted statistically. In other words, it is the statistical properties, which can be obtained by some sort of spatial, temporal or ensemble average, that may appear time-independent. It is also known that the tendency terms $\partial u / \partial t$, $\partial v / \partial t, \partial w / \partial t$ comprise the effects of advection and evolution. For the former, it is possible to account for its influence by applying the concept of a moving frame of reference, as discussed by Gal-Chen (1982). However, the only way to mitigate errors due to evolution is to conduct fast radar volume scans so that the tendency terms can be estimated accurately.

\subsection{Sensitivity to Contaminated Datasets}

Experiment 5 is designed to study the impact of using imperfect data sets, which can be obtained by superimposing random errors of significant magnitudes onto $F, G$ and $H$. In order to reduce the noise, filtering can be applied either on the input data ( $a$ priori) or on the resulting thermodynamic fields ( $a$ posteriori). The latter was advocated by GC when the artificial model-produced data were used for investigation, since he argued that filtering the input data would remove not only the noise, but useful signals as well. Therefore, he suggested applying the filtering only to the results, not the input. However, when GC was applied to a real boundary layer case study (PHOENIX project 1978) in Gal-Chen and Kropfli (1984), a somewhat different conclusion was addressed that the smoothing should be applied not only before, but also after the retrieval was completed. This is called "double smoothing" in their paper. In this 
research, we employ this conclusion.

To prepare the input data sets, let $A$ represent either the $F$, or $G$, or $H$ field at each grid point; it is then superimposed by a random number as shown in the following:

$$
A(i, j, k)=A(i, j, k)+\varepsilon \times(2 \times r d n-1) \times \mathrm{A}(i, j, k),
$$

where " $r d n$ " denotes a random number generator, whose range is from 0 to 1 , and $\varepsilon$ specifies the maximum magnitude of the perturbations to imitate the observational errors. Then, the perturbed field is smoothed by a three-dimensional filter which has the following form:

$$
\bar{A}(i, j, k)=\sum_{l=1}^{1} \sum_{m=-1}^{1} \sum_{n=-1}^{1} w t(i+l, j+m, k+n) \times A(i+l, j+m, k+n),
$$

where $w t$ is the weighting coefficient assigned to the points surrounding the center $(i, j, k)$. For each grid point, a total of 27 points are involved in smoothing the variable. Let $S=|l|+|\mathrm{m}|$ $+|n|$, then when $\mathrm{S}=0$ (the center point), $w t=1 / 8$; when $S=1$ ( 6 points), $w t=1 / 16$; when $S=$ 2 (12 points), $w t=1 / 32 ; S=3$ ( 8 points), $w t=1 / 64$. In order to study the performance of this filter, the low-pass smoothing constraints represented by $P_{5}$ and $P_{6}$ in (6) are turned off in this experiment.

Let the maximum magnitude of the random error $(\varepsilon)$ be $25 \%$ of the value at each point, the statistics in Table 1 demonstrates that SCC scores remain high $(0.895 \sim 0.960)$, while the value of $E_{r}(=0.188)$ indicates that useful information has been extracted through the retrievals.

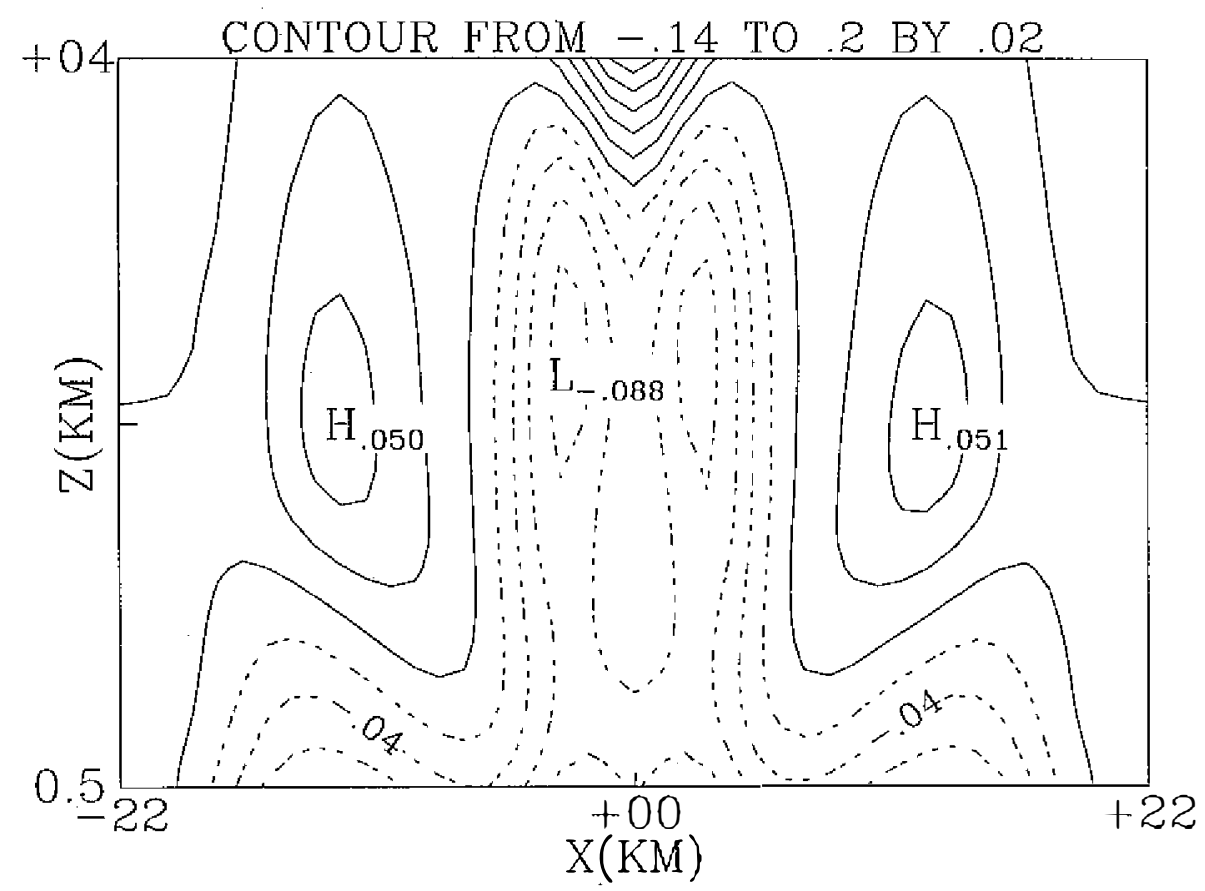

Fig. 15. As in Fig. 1, but for Experiment 5 with input data sets contaminated by randomly perturbed errors. $\mathrm{SCC}=0.971$. 


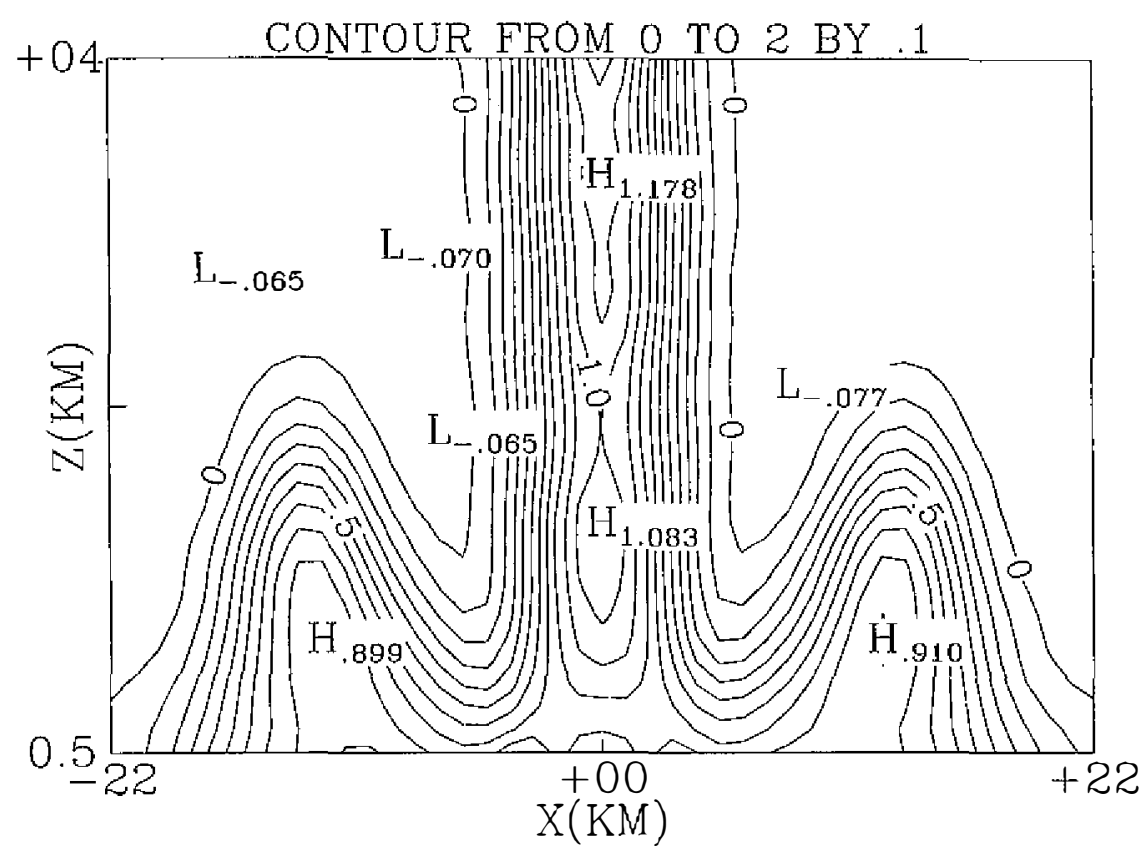

Fig. 16. As in Fig. 2, but for Experiment 5 with input data sets contaminated by randomly perturbed errors. $\mathrm{SCC}=0.986$.

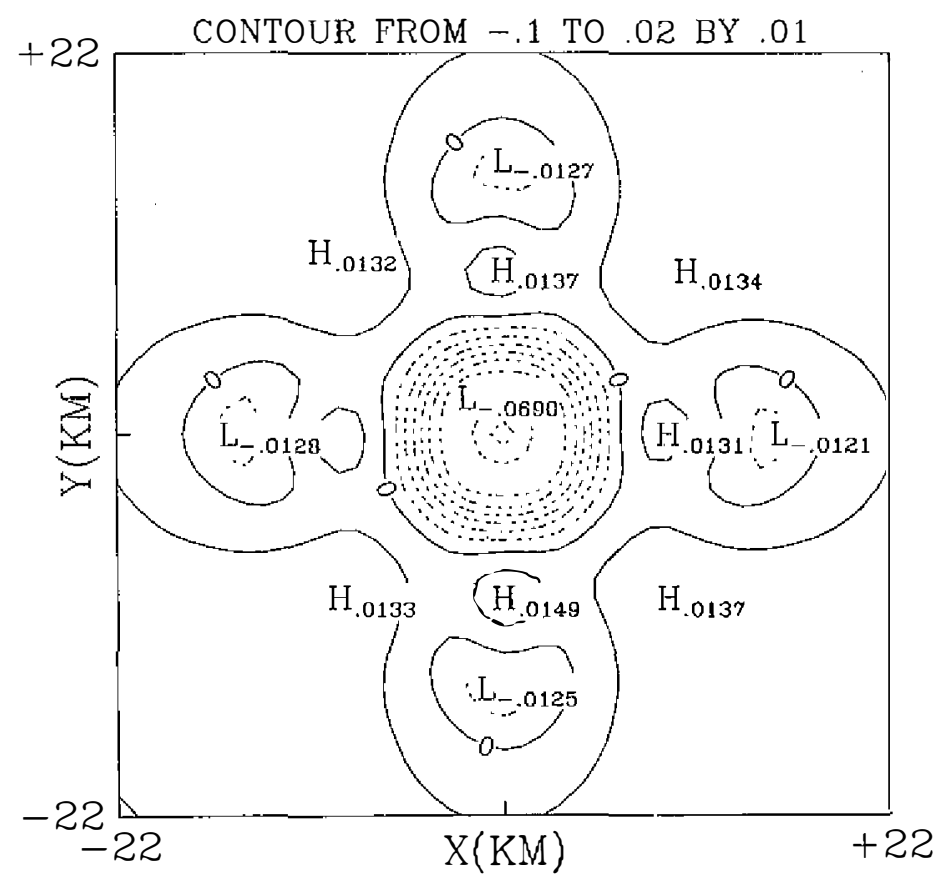

Fig. 17. As in Fig. 3, but for Experiment 5 with input data sets contaminated by randomly perturbed errors. $\mathrm{SCC}=0.998$. 


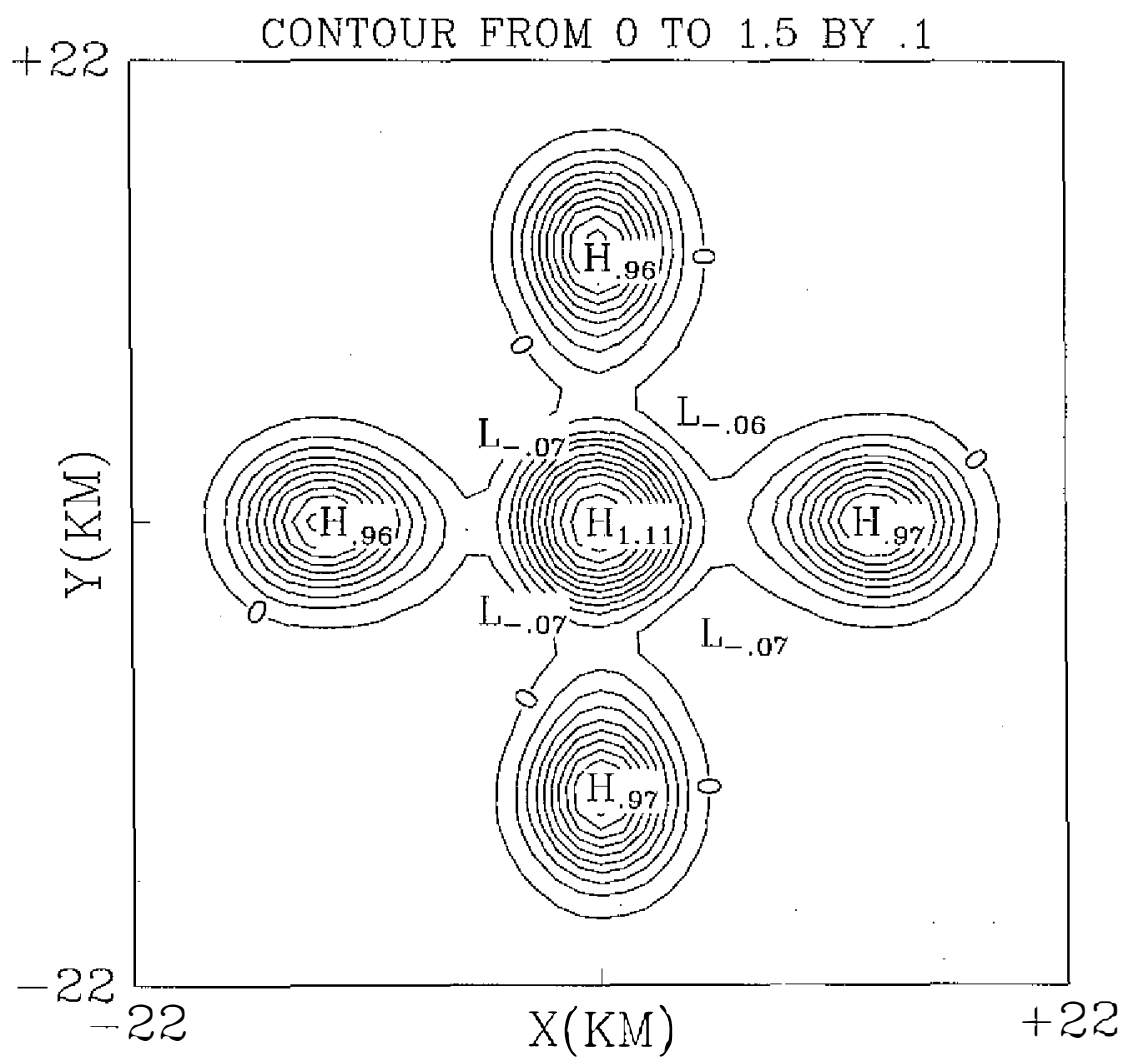

Fig. 18. As in Fig. 4, but for Experiment 5 with input data sets contaminated by randomly perturbed errors. $\mathrm{SCC}=0.998$.

Finally, Figs 15-18 display the vertical and horizontal structures of the retrieved pressure and potential temperature fluctuations respectively for this experiment. The consistency with the "true" solutions displayed in Figs. 1-4 is very encouraging.

\subsection{Application to Topography}

In a mountainous area such as the island of Taiwan, understanding how a weather system (e.g., typhoon, Mei-Yu front, squall line, etc.) evolves and interacts with the topography is always an important issue. To accomplish this goal, it turns out to be a necessary task to conduct thermodynamic retrievals for a non-flat region. Instead of reformulating the governing equations on a terrain-following coordinate system, which is usually the case for doing numerical model simulations, the method proposed in this research can easily adopt the topography by setting the gradients of the cost function $(J)$ with respect to the unknowns $\left(\pi^{\prime}\right.$ and $\left.\theta^{\prime}\right)$ to zeros at these points where the altitudes are below the topography. Experiment 6 demonstrates an example in which the retrieval scheme is utilized in a domain with an idealized bell shaped mountain. The peak of the mountain reaches $900 \mathrm{~m}$, and the half-widths is $10 \mathrm{~km}$. 


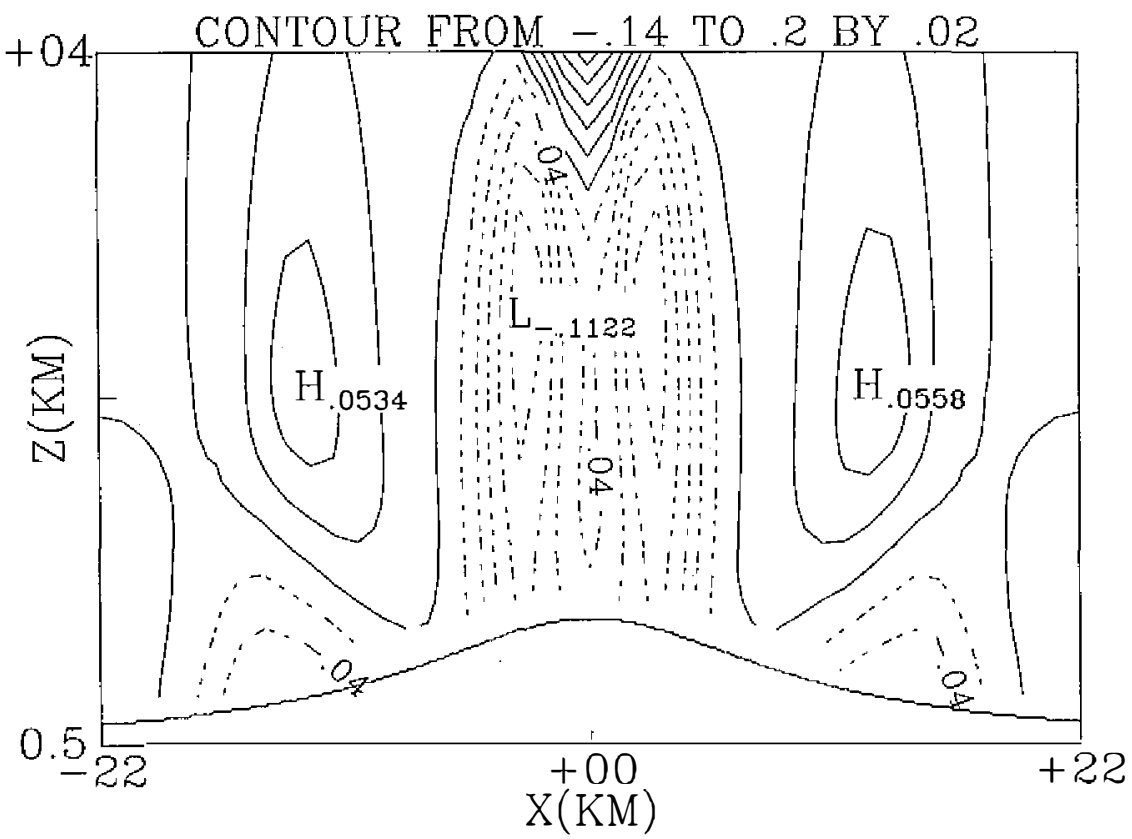

Fig. 19. As in Fig. 1, but for Experiment 6 with topography.

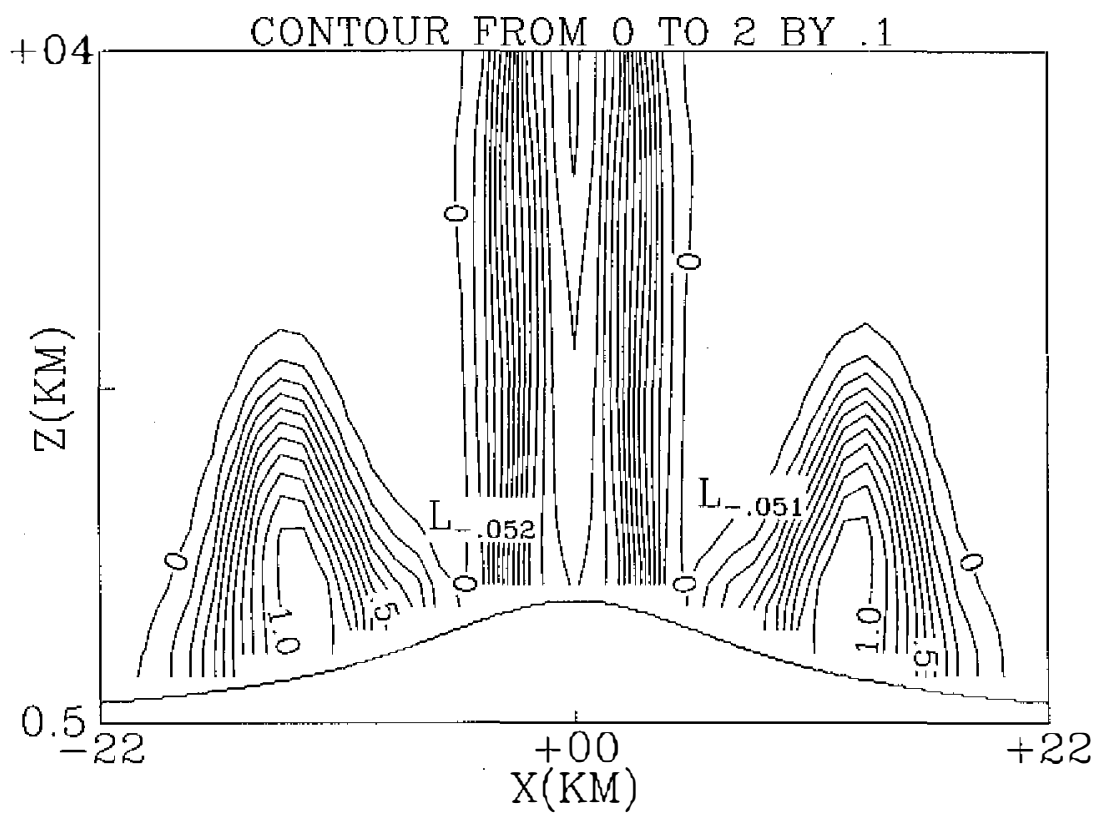

Fig. 20. As in Fig. 2, but for Experiment 6 with topography. 
Figures 19-20 depict the retrieved vertical pressure and potential temperature distributions for a $\mathrm{x}-\mathrm{z}$ cross-section. It can be seen that the positions of the high/low pressure centers, and the structures of the triple thermal bubbles, are all successfully recovered above the terrain.

\section{CONCLUSIONS AND FUTURE WORK}

A different thermodynamic retrieval scheme is developed in this research to extract the pressure and potential temperature field information from 3-D wind observations. The latter, in real cases, can be derived by Doppler radar. Based on the aforementioned results, the following is a list of the conclusions:

- Numerical experiments have shown that the algorithm outlined in this research is a feasible tool to perform thermodynamic retrievals with sufficient accuracy.

- The proposed method can tolerate the errors introduced by using finite differences to approximate the local temporal derivatives, and extract meaningful results from seriously contaminated input data sets, provided an appropriate filter is applied. However, completely neglecting the time rate of change of the wind fields leads to unsuccessful retrievals.

- The retrieval scheme can easily be applied to a region with topography.

- Only 3-D wind data are needed for the retrievals, and the products of the proposed scheme are the three-dimensional potential temperature fluctuations, and the pressure perturbation gradients, in any direction. Therefore, the interpretation of the thermodynamic structures in the vertical direction should no longer cause confusiion. We believe that this is one advantage over the traditional scheme of GC, and should be of particular usefulness in many meteorological applications.

The current data sets focus on simulated data sets, and only preliminary tests are conducted. Future work, some already underway, should include more investigations using Doppler radar observations obtained from real field experiments, and extend the method to incorporate the thermodynamic equation. It is believed that this equation can provide additional information to constrain the gradients of the potential temperature in a three dimensional space.

Acknowledgements The discussion about the retrieval problems with Prof. Tai-Chi Chen is highly appreciated. The author thanks the insightful comments provided by three anonymous reviewers. This work is sponsored by the National Science Council of Taiwan, Republic of China, under Grants NSC 88-2111-M-008-027-A10, NSC 89-2111-M-008-012-A10, and NSC89-2111-M-008-062.

\section{REFERENCES}

Armijo, L., 1969: A theory for the determination of wind and precipitation velocities with Doppler radars. J. Atmos. Sci., 26, 570-573.

Gal-Chen, T., 1978: A method for the initialization of the anelastic equations: Implications for matching models with observations. Mon. Wea. Rev., 106, 587-606. 
Gal-Chen, T., 1979: A method for calculating temperature, pressure and vertical velocities from Doppler radar observations, Preprints, 9th Severe Storms Conference, Kansas City. Amer. Meteor. Soc., 492-496.

Gal-Chen, T., 1982: Errors in fixed and moving frame of reference: Application for conventional and Doppler radar analysis. J. Atmos. Sci., 39, 2279-2300.

Gal-Chen, T., and R. A. Kropfli, 1984: Buoyancy and pressure perturbations derived from Dual-Doppler radar observations of the planetary boundary layer: Applications for matching models with observations. J. Atmos. Sci., 41, 3007-3020.

Hane, C. E., R. B. Wilhelmson, and T. Gal-Chen, 1981: Retrieval of thermodynamic variables within deep convective clouds: Experiments in three dimensions. Mon. Wea. Rev., 109, 564-576.

Hane, C. E., and P. S. Ray, 1985: Pressure and buoyancy fields derived from Doppler radar data in a tornadic thunderstorm. J. Atmos. Sci., 42, 18-35.

Laroche, S., and I. Zawadzki, 1994: A variational analysis method for retrieval of three-dimensional wind field from single-Doppler radar data. J. Atmos. Sci., 51, 2664-2682.

Lin, Y. J., T. C. Wang, and J. H. Lin, 1986: Pressure and temperature perturbations within a squall-line thunderstorm derived from SESAME dual-Doppler data. J. Atmos. Sci., 43, 2302-2327.

Liou, Y.-C., 1999: Single radar recovery of cross-beam wind components using a modified moving-frame of reference technique. J. Atmos. Oceanic Technol., 16, 1003-1016.

Liu, D. C., and J. Nocedal, 1989: On the limited memory BFGS method for large scale optimization. Math. Programming, 45, 503-528.

Parsons, D. B., C. G. Mohr, and T. Gal-Chen, 1987: A severe frontal rainband. Part IIr: Derived thermodynamic stucture. J. Atmos. Sci., 44, 1615-1631.

Qiu, C.-J., and Q. Xu, 1992: A simple adjoint method of wind analysis for single-Doppler data. J. Atmos. Oceanic Technol., 9, 588-598.

Ray, P. S., C. L. Ziegler, and W. Bumgamer, 1980: Single- and Multiple-Doppler radar observations of tomadic storms. Mon. Wea. Rev., 108, 1607-1625.

Roux, F., 1985: Retrieval of thermodynamic fields from Multiple-Doppler radar data using the equations of motion and the thermodynamic equation. Mon. Wea. Rev., 113, 21422157.

Roux, F., 1988: The West African squall line observed on 23 June 1981 during COPT 81: Kinematics and thermodynamics of the convective region. J. Atmos. Sci., 45, 406-426.

Roux, F., and J. Sun, 1990: Single-Doppler observations of a West African squall line on 27 28 May 1981 during COPT 81: Kinematics, thermodynamics and water budget. Mon. Wea. Rev., 118, 1826-1854.

Roux, F., V. Marecal, and D. Hauser, 1993: The 12/13 January 1998 Narrow Cold-Frontal Rainband Observed during MFDP/FRONTS 87. Part I: Kinematics and Thermodynamics. J. Atmos. Sci., 50, 951-974.

Shapiro, A., S. Ellis, and J. Shaw, 1995: Single-Doppler velocity retrievals in the planetary boundary layer. J. Atmos. Sci., 52, 1265-1287.

Sun, J., D. W. Flicker, and D. K. Lilly, 1991: Recovery of three-dimensional wind and tem- 
perature fields from simulated Doppler radar data. J. Atmos. Sci., 48, 876-890.

Sun, J., and N. A. Crook, 1996: Comparison of thermodynamic retrieval by the adjoint method with the traditional retrieval method. Mon. Wea. Rev., 124, 308-324.

Testud J., and M. Chong, 1983: Three-Dimensional Wind Field Analysis form Dual-Doppler Radar Data. Part I: Filtering, Interpolating and Differentiating the Raw Data. J. Climate and Applied Meteo., 22, 1204-1215. 